Abstract C-C3-04

Changing Prescriber Behavior Using Academic Detailing and Computerized Decision Support

Steven R. Simon, MD, MPH, Harvard Pilgrim Health Care; Robert J. Fortuna, MD, Harvard Pilgrim Health Care; Francis X. Campion, MD, FACP, Harvard Vanguard Medical Associates; Adrianne C. Feldstein, MD, Kaiser Permanente Northwest; David H. Smith, PhD, Kaiser Permanente Northwest; Marvin Packer, MD, Harvard Vanguard Medical Associates; Brent Cerullo, Harvard Vanguard Medical Associates; Fang Zhang, $\mathrm{PhD}$, Harvard Pilgrim Health Care; Dennis Ross-Degnan, ScD, Harvard Pilgrim Health Care; Jonathan A. Finkelstein, MD, MPH, Harvard Pilgrim Health Care

Background/Aims: Harvard Pilgrim Health Care (HPHC) is one of 28 Attorney General Consumer and Prescriber Grant Program grantees. Four HMORN CERTs sites, including HPHC, received grants. An overall goal of the Program is to identify best practices for educating clinicians about the influences on their prescribing and for changing their prescribing behaviors. At HPHC, our aim was to test the effectiveness of computerized clinical decision support (alerts), alone and in combination with group academic detailing, to reduce the use of heavily marketed hypnotic medications at Harvard Vanguard Medical Associates (HVMA), a 14-site group practice that uses the Epic electronic health record. Methods: We randomly allocated the 14 practices to three study groups: (1) Alerts Only ( $\mathrm{n}=5$ practices), (2) Alerts plus Detailing $(\mathrm{n}=5)$, and (3) Usual Care $(\mathrm{n}=4)$. Internal medicine clinicians in both the Alerts groups received prescribing alerts when initiating new prescriptions for eszopiclone (Lunesta), zaleplon (Sonata), ramelteon (Rozerem), and controlled-release zolpidem (Ambien CR) between March and December 2007. We carried out group academic detailing sessions in June and July 2007 followed by an educational mailing in the fall. Results: A pre-planned interim data analysis (August 2007) demonstrated that hypnotic study alerts were triggered on 98 separate occasions. After receiving an alert, $30 \%(95 \% \mathrm{CI}, 21 \%-40 \%)$ of clinicians changed the prescription to an alternative recommended medication. We plan to extract post-intervention data in January 2008 and will present findings of the intervention at the HMORN meeting. In addition, we carried out pre-intervention and post-intervention surveys of all clinicians at HVMA in 2006 and 2007 which assessed clinicians' perceptions of the influences on their prescribing, with special attention to the role of direct-to-consumer marketing. We will present the results of these surveys, as well. Conclusions: Developing interventions using health information technology and group detailing to improve the use of medications is a high priority for many HMORN organizations. The results of this study should be generalizable to many HMORN sites, including but not limited to the many organizations that have deployed the Epic electronic health record.

Abstract C-C3-05

Electronic Support for Public Health (ESP): Automated Detection and Reporting of Notifiable Conditions

Michael Klompas, MD, MPH, Harvard Pilgrim Health Care; Ross Lazarus, MBBS, MPH, Channing Laboratory, Brigham and Women's Hospital; Gillian A. Haney, MPH, Massachusetts Department of Public Health; Xuanlin Hou, MSc, Channing Laboratory, Brigham and Women's Hospital; Dumas William, RN, Massachusetts Department of Public Health; Francis X. Campion, MD, Atrius Health; Benjamin A. Kruskal, PhD, Atrius Health; Alfred DeMaria, MD, Massachusetts Department of Public Health; Richard Platt, MD, MSc, Harvard Pilgrim Health Care and Harvard Medical School

Background: Legislation and good citizenship compel practitioners to report key diagnoses that affect the public good. Public health reporting, however, is frequently paper-based, incomplete, lacking key details, and delayed. We sought to leverage electronic medical record (EMR) systems to automate the detection and reporting of notifiable diseases to a health department. Methods: The Electronic Support for Public Health (ESP) system consists of an independent server within the central data processing center of Atrius Health, a large multisite medical practice with over 600,000 patients. The server is populated by nightly extracts generated by Atrius Health's Epic Care EMR. The extracts contain codified data on every patient encounter from the preceding 24 hours including demographics, diagnoses, lab orders, lab results, vital signs, vaccinations, and prescriptions. Relevant codes are mapped to standard nomenclatures. EMR data on the server is analyzed using novel algorithms to detect patients with notifiable conditions. When a patient is identified, an HL7 electronic message is securely transmitted to the state health department. The ESP server is deliberately decoupled from the source EMR in order to minimize interference with clinical computing, ease modification of case-detection algorithms, and permit compatibility with different proprietary EMR systems. The number and completeness of electronic case reports are compared to concurrent, conventional, paper-based reports. Results: ESP has been operational since January 2007 and has reported 1121 cases of chlamydia, 146 cases of gonorrhea, 25 cases of pelvic inflammatory diseases, and five cases of acute hepatitis A. Comparison with ongoing traditional reporting shows a $44 \%$ increase in the number of chlamydia and gonorrhea cases reported. ESP includes treatment details on $100 \%$ of reports versus $88 \%$ of manual reports and has noted 86 cases of concurrent pregnancy versus five in paper reports. Algorithms to detect additional disease are being validated. Conclusions: Automated analysis of electronic medical record data can increase the number, clinical detail, and accuracy of notifiable disease reporting. The architecture pioneered for this system can potentially support additional public health objectives such as novel vaccine or medication adverse event detection and reporting. Collaborators are invited to help refine the system and test implementation in a new site.

\section{Abstract C-D2-01 \\ The Use of Computerized Medical Records to Investigate the Association Between Diabetes and End Stage Renal Failure}

Varda Shalev, MD, MHA, Maccabi Healthcare Services; Gabriel Chodick, $\mathrm{PhD}$, Maccabi Healthcare Services, Sackler Faculty of Medicine, Tel Aviv University, Tel Aviv, Israel; Ehud Kokia, MD, Maccabi Healthcare Services; Anthony D. Heymann, MD, BSc, MB, BS, MRCGP, MHA, Maccabi Healthcare Services, Sackler Faculty of Medicine, Tel Aviv University, Tel Aviv, Israel

Background: In many countries diabetes-mellitus (DM) is a leading cause for end-stage renal-failure (ESRF). The objective of the current study is to demonstrate the use of computerized medical records to describe the association between the prevalence of ESRF patient needing dialysis and DM by using data from both types of patient populations. Methods: Data from an Israeli HMO were used to identify 57,030 DM-patients and 647 ESRF-patients. Nearly one-third (30.8\%) of the ESRF patients had DM. Results: ESRF-patients $<35$ years had a 45 -fold higher prevalence of DM compared to the general population. Overall, ESRF women had a weighted prevalence rate of 3.11 (95\% CI, 2.63-3.68) for diabetes; a significantly lower prevalence rate of 2.03 was calculated for men ( $95 \%$ CI, 1.75-2.36; $P<0.05)$. Conclusions: The present study demonstrates the potential of harnessing large computerized medical databases to provide epidemiological data about the association of important chronic diseases which can be used to explore and improve the quality of disease management of DM and ESRF patients, and for health service planning and research.

\section{Abstract C-D2-03 \\ Family Health History: New Standards and Enhanced Utility for a Valuable Preventive Tool}

Mary Beth Bigley, DrPH, MSN, Office of the US Surgeon General, Health and Human Services; Feero Greg, MD, PhD, National Human Genome Research Institute, National Institutes of Health; Downing Gregory, MD, $\mathrm{PhD}$, Personalized Health Care Initiative, Health and Human Services

Background: Family health history $(\mathrm{FH})$ is an underutilized tool in healthcare and could play a central role in enhancing the use of preventive services for a variety of disorders of major public health importance. Existing clinical prevention guidelines for screening and management of disorders incorporate $\mathrm{FH}$ information. Unfortunately, obtaining a $\mathrm{FH}$ is time-consuming and many primary care providers are insufficiently trained to effectively interpret the information they obtain. Studies show that clinicians often neglect FH in the context of healthcare visits. A potential solution would be the development and widespread adoption of interoperable health information technology (HIT) systems that facilitate patient entry of FH information and provide automated clinical decision support for 
clinicians. Methods: The American Health Information Community (AHIC) is a federal advisory body organized to make recommendations to the Secretary of the US Department of Health and Human Services on how to accelerate the adoption of HIT. In July 2007, AHIC advanced recommendations to improve electronic health information exchange via the implementation of standards for FH information. The first step to developing interoperable HIT systems for the collection, interpretation, and exchange of $\mathrm{FH}$ information is the development of a widely accepted core minimum dataset for FH. To this end, the Personalized Healthcare Workgroup of the AHIC assembled the FH Multi-Stakeholder Workgroup to develop a minimum core dataset. The workgroup is comprised of over 40 members representing approximately 18 different public and private organizations with expertise in the area of $\mathrm{FH}$, HIT, and healthcare delivery. Results: The FH core dataset for primary care was developed using an interactive, iterative process that used the proposed standards for FH information contained in the Continuity of Care Record (CCR) as a foundational document. Some key features of the core dataset are, FH information should be collected in a structured manner that permits the generation of a pedigree, age of disease onset should be collected, and selfidentified race and ethnicity data should be included as FH information. The document outlining the requirements will be made available for public comment in early 2008. Conclusions: The document should serve as a solid foundation for public and private efforts to create HIT systems and demonstration projects examining the utility of interoperable $\mathrm{FH}$ information in clinical and research settings.

\section{Abstract C-D2-05 \\ Creation of State Legislation to Protect and Facilitate Use and Exchange of Electronic Health Information}

Margaret J. Gunter, PhD, Lovelace Clinic Foundation; Shelley Carter, RN, MPH, MCRP, Lovelace Clinic Foundation; Bob Mayer, New Mexico Department of Health; Randall McDonald, JD, Miller Stratvert P.A.

Background: In examining potential barriers to implementing a statewide health information exchange in NM, we identified as a key barrier NM's laws regarding the protection of health information. The laws are fragmented and dated, adopted prior to HIPAA, and address only health data in paper-based formats. Enactment of specific state legislation authorizing transmission, use, and exchange of electronic health information would eliminate the detrimental effects of the existing state laws. Aim: To develop and pass comprehensive state privacy legislation in New Mexico governing the use, storage, and exchange of electronic health information. Methods: Review of health privacy legislation nationally, interviews with privacy experts, development of a draft of the legislation, and solicitation of input from a variety of stakeholder groups to identify controversial areas of the legislation and to garner legislative support were performed. Key privacy experts (e.g., legislators, healthcare providers, and attorneys) discussed their concerns and opinions about electronic transmission of health information. Attorneys researched privacy legislation in other states and drafted NM's Electronic Health Information Act. This draft was examined and discussed with numerous stakeholder groups to determine needed revisions. Results: Interviews with key privacy experts and stakeholders revealed conflicting points of view concerning the legislation, especially regarding issues of patient consent. Patient privacy advocates strongly favored patient control of access to their health data and more restrictive rules for patient consent than required by HIPAA. In contrast, providers, payers, and advocates for health information exchange networks typically favored less restrictive patient consent (consistent with current HIPAA regulations) to avoid additional time, burden, expense, and delays in implementing health information exchange. Review of legislation in other states revealed only Minnesota and Nevada have enacted laws specifically addressing electronic health information exchange. Conclusions: Current NM legislation (and legislation in most other states) does not adequately address the use and exchange of electronic health information. Current efforts to develop health information exchange networks in NM and elsewhere require resolution of key patient privacy issues and the passage of appropriate legislation. Funded by the Agency for Healthcare Research and Quality.

\section{Health Economics}

\section{Abstract C-C4-01 \\ Prevalence-Based Costs of HIV-Positive Patients With Multiple HAART Switches}

Richard T. Meenan, PhD, MPH, MBA, Kaiser Permanente Center for Health Research; Michael A. Horberg, MD, MAS, FACP, Division of Research, Kaiser Permanente Northern California; Maureen C. O'Keeffe-Rosetti, MS, Kaiser Permanente Center for Health Research; Terry Kimes, MS, Kaiser Permanente Center for Health Research; Wendy Leyden, MS, Division of Research, Kaiser Permanente Northern California; Diana Antoniskis, MD, Immune Deficiency Clinic, Kaiser Permanente Northwest

Background/Aims: Highly active antiretroviral therapy (HAART) successfully treats patients with HIV. However, many patients do not achieve complete suppression. Perhaps the most important reason for treatment failure is infection with antiretroviral drug-resistant variants, which threatens to increase treatment cost as well as limit HAART's clinical effectiveness. This study examines the resource utilization and cost implications of multi-drug resistance using the HIV+ population of Kaiser Permanente Northern California (KPNC). Methods: Retrospective cohort analysis of all HIV+ patients in KPNC between January 2000 and June 2005 (total study period). Continuous active membership of 12 months and minimum 12 months of continuous HAART. Cases defined as HIV+ patients on third or later HAART regimen; controls defined as HIV+ patients on first or second HAART regimen. Regimen switch defined as combination change of two or more additions or subtractions of antiretroviral therapy (ART) drugs to or from an existing HAART regimen. Costs include outpatient, inpatient, and pharmacy categories. Results: All results are for 2004 only (291 cases, 1,676 controls); costs reported in person-years. Mean total pharmacy costs were $\$ 17,668$ for cases vs $\$ 12,417$ for controls $(P<0.04)$. Of mean pharmacy costs for cases, $85 \%$ were ART-based (controls $=90 \%$ ). Mean number of 2004 fills for both ART and non-ART drugs were higher for cases (20 vs 15 for ART; 21 vs 15 for non-ART). Mean total outpatient costs were $\$ 3,275$ for cases vs $\$ 2,238$ for controls $(P<0.06)$. Mean ER visits and mean primary care visits, but not specialty care visits, were both higher for cases than for controls $(P<0.03)$. Mean inpatient costs were $\$ 3,803$ for cases vs $\$ 1,738$ for controls. Mean number of stays was higher for cases than for controls ( 0.29 vs 0.16 ; $P<0.05$ ), but length of stay did not differ between groups. Conclusions: In 2004, patients with HIV on at least their third HAART regimen incurred mean pharmacy costs $40 \%$ higher than similar patients on first or second regimen. Outpatient and inpatient costs also appear higher among cases, but not strikingly so. Overall cost differentials between cases and controls may be underestimates, as laboratory, radiology, and home health have not yet been added. These cost categories may well widen the cost differences between HIV+ patients who have been forced to switch HAART regimens more than once relative to those who have not.

\section{Abstract C-C4-04 \\ Entering and Exiting the Coverage Gap by Medicare Part D Beneficiaries With Diabetes: a TRIAD Study}

Julie A. Schmittdiel, $\mathrm{PhD}$, Kaiser Permanente Northern California Division of Research; Susan L. Ettner, PhD, University of California Los Angeles; Vicki Fung, PhD, Kaiser Permanente Northern California Division of Research; Jie Huang, PhD, Kaiser Permanente Northern California Division of Research; Norman Turk, MS, University of California Los Angeles; Elaine Quiter, MS, University of California Los Angeles; Bix Swain, MS, Kaiser Permanente Northern California Division of Research; John Hsu, MD, MBA, MSCE, Kaiser Permanente Northern California Division of Research; Carol M. Mangione, MD MPH, University of California Los Angeles

Background/Aims: Many Medicare Part D enrollees face gaps in their prescription drug cost coverage after $\$ 2,250$ in annual drug expenditures. Beneficiaries with chronic illnesses may be particularly vulnerable to coverage gaps, with estimates ranging as high as $64 \%$ of diabetic beneficiaries entering the gap. We examined total drug costs and out-of-pocket drug costs, and rates of entry and exit into the Part D coverage gap for beneficiaries with diabetes in two large California Medicare Advantage plans. Methods: Beneficiaries from two large health plans in the TRIAD (Translating Research Into Action for Diabetes) Study offering Medicare Advantage Part 